their years of study. The book contains very extensive notes and references at the end of each chapter. It is a volume well worth having on one's bookshelf.

Hugo STEINFINK

Department of Chemical Engineering

Materials Science and Engineering

University of Texas

Austin

TX 78712

USA

Acta Cryst. (1994). A50, 797

Random, non-random and periodic faulting in crystals. By M. T. Sebastian and P. Krishna. Pp. xv + 383. Reading: Gordon and Breach Science Publishers, 1994. Price £85.00. ISBN 2-88124-925-6.

This book extends considerably a previous useful effort: Polymorphism and polytypism in crystals (A. R. Verma and P. Krishna, 1966). The new title promises a general treatment of faulted crystals, but discussion focuses mainly on layer structures. The book contains five chapters: introduction, stacking faults in close-packed structures, diffuse X-ray scattering from randomly faulted close-packed structures, phase transformations and non-random faulting in close-packed structures, and periodic faulting in crystals: polytypism.

Although the authors face the obstacle of the absence of a single comprehensive theory of faulting, they succeed in achieving a useful result. I found it a real pleasure to peruse this well written book by credentialed experts. Besides their own early and significant studies, the authors cite nearly 1100 references, although, surprisingly, they omit an important early theoretical study by Landau and Lifshitz in 1937.

Chapters 3 and 4 , on diffuse X-ray scattering and phase transformations, comprise the new book's heart and about $60 \%$ of its length. Except, perhaps, for some dusty unpublished doctoral thesis sitting somewhere, these chapters are the most thorough existing treatment of these topics.

My principal disappointment with the book is its less than thorough treatment of what is arguably the single most important faulting parameter: stacking-fault energy $\gamma$. The authors provide little description of how $\gamma$ changes with composition, pressure or temperature; nor of how $\gamma$ relates to many solid-state phenomena. They fail to tabulate two closely related properties: surface energy and twin-boundary energy, and they almost ignore the tremendous recent strides in calculating $\gamma$ from theory, using more powerful computers and better computational models.

However, I like the book and find it correct and useful. I like its basic setup: author and subject indexes, good accessibility, lots of diagrams and figures and sensible organization. Anyone, from undergraduates to advanced researchers, who is fascinated by crystalline faults will enjoy consulting this book and will profit from the authors' expertise. Also, I share the view, expressed by the authors in their concluding remarks, that we should keep an Aristotelian, non-Baconian approach to science. We should pursue the mystery, savor the knowledge and treat applications as byproducts. That $A B X_{3}$ perovskites and many of the new perovskite-based high- $T_{c}$ oxide superconductors show polytypic structures is perhaps application enough!

\section{HASSEL LEDBETTER}

Materials Science and Engineering Laboratory

National Institute of Standards and Technology

Boulder

CO 80303

USA

Acta Cryst. (1994). A50, 797

\section{Books Received}

The following books have been received by the Editor. Brief and generally uncritical notices are given of works of marginal crystallographic interest; occasionally, a book of fundamental interest is included under this heading because of difficulty in finding a suitable reviewer without great delay.

Band theory of solids: an introduction from the point of view of symmetry. By S. L. AlTMANN. Pp. xiv + 286. Oxford: Oxford Science Publications (OUP), 1994. Price $£ 19.50$ (paperback). ISBN 0-19-855866-X. This is a reissue, in paperback and with corrections, of the author's original 1990 book of the same title. The original work was designed to 'bridge the gap between the current elementary texts on band theory and the more advanced treatments of the group theory of solid state'. Though intended to appeal to all physical scientists, the book is directed mainly at chemists. 